\title{
GALACTIC AND EXTRAGALACTIC APPLICATIONS
}

\author{
G.B. FIELD \\ Harvard-Smithsonian Center for Astrophysics \\ Cambridge, MA 02138, U.S.A.
}

For the purposes of this talk I will take "high resolution" to mean 1 milliarcsecond (mas). Only objects of high surface brightness when averaged over 1 mas are considered; as H. McAlister will discuss stellar applications, I will speak only about nonstellar applications.

Interesting nonstellar objects in the Galaxy include disks in binary systems, in protostellar systems, and in the galactic center. Unfortunately, accretion disks in X-ray binaries are probably too small to be resolved, about 0.03 mas in CygX-1. A protostellar disk in Orion at 400 pc could be resolved if its diameter exceeds $0.4 \mathrm{AU}$; as the temperature is low, this requires infrared or radio observations. At the distance of the galactic center, 1 mas corresponds to $10 \mathrm{AU}$; if there is a black hole of $10^{6} \mathrm{M}_{\odot}$, the material in its accretion disk would have a velocity at that distance of $10,000 \mathrm{~km} \mathrm{~s}^{-1}$ and a dynamical time scale of the order of a day. VLBI observations have already shown that the radio source is about $10 \mathrm{AU}$ across. Infrared or longer wavelengths are necessary to penetrate the extinction.

An angular resolution of 1 mas at optical wavelengths would permit new studies of stellar kinematics, as a proper motion of $1 \mathrm{mas} / \mathrm{y}$ corresponds to $150 \mathrm{~km} \mathrm{~s}^{-1}$ at $30 \mathrm{kpc}$. The proper motions of selected stars in globular clusters would make it possible to derive the space motions of globular clusters and hence to constrain models of the galactic gravitational field at substantial galactocentric distances. Imaging at the 1 mas level would reveal stars in the cores of globular clusters without confusion.

At the distance of M31, 1 mas corresponds to $0.004 \mathrm{pc}$, sufficient to probe concentrations of stars at the center of M31 or its companions. At the Virgo cluster 1 mas corresponds to $0.05 \mathrm{pc}$, about 1/10 of the upper limit (from VLBI) for the sizes of hot spots in the jet of M87. As the hot spots could be sources of energetic electrons (we know from lifetime arguments that electrons don't propagate all the way from the central engine), it would be interesting to study them both in the optical and with the improved dynamic range to be expected in the radio with VLBA. High-resolution optical emission line studies might reveal whether the gas in a cooling flow at the center of M87 forms stars or falls further into the nucleus, perhaps feeding the black hole believed to be there.

Supernovae occur often in the Virgo cluster, and a light echo from a supernova travels $0.3 \mathrm{pc}=1$ mas per year. Study of such echoes would provide a way to estimate the distance of Virgo.

Seyferts, about $1 \%$ of all galaxies, contain active nuclei that are fainter than quasars. At the $10 \mathrm{Mpc}$ distance of the Seyfert galaxy NGC 4151, 1 mas corresponds to two light months. As its optical light varies on a time scale of months, observations could help to clarify the mechanism responsible. At the quasar $3 \mathrm{C} 273$, whose redshift is $0.15,1$ mas 
corresponds to $10 \mathrm{ly}$ in a $q_{0}=1 / 2$ universe with $H_{0}=50$. Light variations occur on the scale of years, and it would be interesting to find out whether the broad emission lines originate at this radius or less in $3 \mathrm{C} 273$.

Radio observations of quasars reveal jets which carry energy from a central engine probably located in the nucleus of a galaxy. At a VLBI resolution of $\sim 1$ mas, jets appear to originate in a high-brightness core which is self-absorbed at centimeter wavelengths. It is important to penetrate into the core with the highest possible angular resolution and at the highest possible frequency. The VLBA, operating at its shortest wavelength, $7 \mathrm{~mm}$, will be able to do this. A preliminary study, carried out with a few of the antennas of the VLBA, has recently achieved a resolution of 0.1 mas on 3 C84 at a wavelength of $7 \mathrm{~mm}$.

Improved dynamical range at high resolution will also bear on the problem of superluminal motion in quasar jets. The model that assumes that superluminal motion is an optical illusion due to nearly luminal motion of a quasar jet directed nearly at the observer and seen more readily because of Doppler boosting, runs into the difficulty that such alignments are too improbable to account for the high incidence of the phenomenon. Perhaps the alternative suggestion, that jets themselves are rarely pointed at the observer, but that energetic acceleration towards the observer occurs here and there within the jet, is more likely. If so, the greater dynamic range of VLBA as compared with previous VLBI experiments will be useful in identifying the features not directed toward the observer, but much fainter because of the lack of Doppler boosting.

As we consider objects of large redshift, such as Lyman- $\alpha$ galaxies with redshifts from 1.6 to 3.8 and quasars with redshifts up to 4.1 , we recall that in relativistic models of the universe, the normalized angular diameter $c \delta / H_{0}$ subtended by an object of size $D$ is $D$ times a function $f(z)$ of redshift $z$ that is $z^{-1}$ for $z \ll 1$ and that flattens out for $z \gtrsim 0.5$. For $q_{0}=0, f(z)$ decreases from 3.8 to 2.0 as $z$ increases from 0.5 to 4 , while for $q_{0}=1 / 2, f(z)$ decreases from 4.3 to a minimum value of 3.4 at $z=1.25$, then increases again to 4.5 at $z=4$. If we adopt $c \delta / H_{0} \sim 3 D, \delta=1$ mas corresponds to $D \sim 10$ pc in these objects. The most distant objects are of great interest because they are being observed at the greatest look-back time. If there is a critical density of cold dark matter in the universe, galaxies are expected to form over the range $z=0$ to 5 , with a peak of activity at $z=3$; this theory can be tested by studying star formation at various redshifts. The Lyman- $\alpha$ galaxy $0902+34$ with a redshift of 3.4 has an exponential dependence of brightness on radius, with characteristics scale 2200 mas $=22 \mathrm{kpc}$, much larger than the high resolution element under discussion. If Lyman- $\alpha$ line is due to many HII regions ionized by hot young stars, this could be tested with a high-resolution image.

Finally, one always hopes that a "standard rod", expected to have the same length in galaxies of all redshifts, will be discovered, as observations of such an object would reveal its angular size, and, when $z$ is known, $f(z)$ as explained above. Comparison with theory yields $q_{0}$. Although no such objects are known now, the enormous increase in resolution in going to 1 mas improves the chances they will be discovered. 